\title{
An Error Estimate for the Truncation Method for the Solution of Parabolic Obstacle Variational Inequalities
}

\author{
By Alan E. Berger* and Richard S. Falk**
}

\begin{abstract}
A rate of convergence is obtained for a truncation method for the numerical solution of a class of parabolic variational inequalities.
\end{abstract}

1. Introduction. The truncation method was originally developed for the numerical solution of a free boundary problem modeling simultaneous diffusion and absorption of a chemical within a substrate [2]. This free boundary problem and a related free interface problem describing the potential in certain semiconductors have been shown to be equivalent to obstacle variational inequalities [1], [7], which may be solved using the truncation method [1]. Problems in fluid dynamics and elastoplastic behavior of materials have been formulated in terms of more general variational inequalities in [4].

The truncation method is a "fixed domain" method, in that it replaces geometrical front tracking (which can become quite complicated in two or three space dimensions) by simple algebraic operations. We will develop an error estimate for a special case of the truncation method for the numerical solution of parabolic obstacle variational inequalities (Theorem 1 below). The rate of convergence corresponds to that found by numerical experiments in [1] and [2]. This will be done by considering the truncation method procedure as a perturbation (due to quadrature errors) of an $L^{2}$ projection type scheme (as presented for example in [9]) for which an error estimate has been obtained [8].

We first present the method under consideration, show how it can be considered as a perturbation of a scheme involving $L^{2}$ projection, and then state and prove the convergence result.

2. The Parabolic Variational Inequality and the Truncation Method. Let $\Omega$ be a smooth convex domain in $R^{2}$ (or $R^{1}$ ) with boundary $\Gamma$. We define $(v, w)=\int_{\Omega} v w d z$ for $v, w \in L^{2}(\Omega)$, and $a(v, w)=\int_{\Omega} \nabla v \cdot \nabla w d z$ for $v, w \in H_{0}^{1}(\Omega)$. Let $\psi \in C^{2}(\bar{\Omega})$, with $\psi \leqslant 0$ on $\Gamma$, and let $f, f_{t} \in C([0, T] \times \bar{\Omega})$. Define

$$
K=\left\{v \in H_{0}^{1}(\Omega) / v \geqslant \psi \text { a.e. (almost everywhere) in } \Omega\right\},
$$

Received July 6, 1976.

AMS (MOS) subject classifications (1970). Primary 35K20, 65N15, 65N30; Secondary $41 \mathrm{A25}$.

Key words and phrases. Variational inequality, truncation method, error estimate.

* Work supported by the Naval Air Systems Command (WR01-402-001) and the Office of Naval Research (NR044-499).

** This research was supported by NSF grant MPS 74-05795 
and let $u^{0}$ be given in $K \cap W_{\infty}^{2}(\Omega)$. The variational inequality to be considered is to find $u(p, t)$ such that

$$
u(p, t) \in K \text { for } t \geqslant 0,
$$

(1b) $\left(u_{t}, v-u\right)+a(u, v-u) \geqslant(f, v-u)$ for all $v \in K$, almost all $t>0$,

$$
u(p, 0)=u^{0}(p) \text { for } p \in \Omega .
$$

From [3], for any fixed $T>0,(1)$ has a unique solution, and $u(\cdot, t) \rightarrow K$ is continuous, $u_{t} \in L^{2}\left(0, T, H_{0}^{1}(\Omega)\right)$, and

$$
\sup _{0 \leqslant t \leqslant T}\left(\|u(\cdot, t)\|_{H^{2}(\Omega)}+\left\|u_{t}(\cdot, t)\right\|_{L^{\infty}(\Omega)}\right)<\infty .
$$

The general truncation method for (1) is [1]: given approximate solution values $U^{n}$ at time $t^{n}$, one uses any appropriate numerical method to obtain the solution $\widetilde{U}^{n+1}$ at $t^{n+1}$ of

$$
\begin{gathered}
u_{t}=\nabla^{2} u+f \text { in } \Omega, \\
u=0 \text { on } \Gamma, \\
u\left(\cdot, t^{n}\right)=U^{n} .
\end{gathered}
$$

Then $U^{n+1}$ at a mesh point $P$ of the numerical scheme being used is defined to be

$$
U^{n+1}(P)=\max \left(\psi(P), \widetilde{U}^{n+1}(P)\right) .
$$

We will consider the situation when (2) is solved using the finite element method with continuous piecewise linear basis functions, explicit time discretization, and "lumping" of the mass matrix. We now give some notation. Let $\Omega_{h}$ be a triangulated polygonal region inscribed in $\Omega$ (with maximum mesh length $h$ which is assumed to be bounded by some constant $C_{1}$ ), and let $P_{1}, \ldots, P_{m}$ be the vertices lying inside $\Omega$. The piecewise linear basis function $\varphi_{j}$ is 1 at node $P_{j}, 0$ at the other vertices, and extended by 0 outside $\Omega_{h}$. We define $S_{h}=\left\{\sum_{j=1}^{m} V_{j} \varphi_{j} / V_{j} \in R^{1}\right\}$ and

$$
K_{h}=\left\{V \in S_{h} / V\left(P_{j}\right) \geqslant \psi\left(P_{j}\right), j=1, \ldots, m\right\} .
$$

Since $\Omega$ has been assumed to be convex, $S_{h} \subset H_{0}^{1}(\Omega)$. We will use the convention that if $V=\Sigma_{j=1}^{m} V_{j} \varphi_{j}$, then $V$ can refer to either the element of $S_{h}$ or the column vector $\left(V_{1}, \ldots, V_{m}\right)$ as the context indicates. The mass matrix $M$ is $M_{i j}=\left(\varphi_{i}, \varphi_{j}\right)$, the stiffness matrix $S$ is $S_{i j}=a\left(\varphi_{i}, \varphi_{j}\right)$, and the load vector $F_{j}^{n}$ is $\left(\varphi_{j}, f(\cdot, n \Delta t)\right)$. The lumped mass matrix $L$ is obtained by computing $\int_{\Omega} \varphi_{i} \varphi_{j} d z$ by the quadrature rules

$$
\begin{aligned}
\int_{a}^{b} g d x & \sim(b-a)(g(a)+g(b)) / 2 \quad\left(\text { in } R^{1}\right) ; \\
\int_{a b c} g d z & \sim(g(a)+g(b)+g(c)) \text { area }(a b c) / 3 \quad\left(\text { in } R^{2}\right) .
\end{aligned}
$$

Then letting $I$ be the $m \times m$ identity matrix, the specific truncation method for which we will obtain a rate of convergence is 


$$
\begin{aligned}
\widetilde{U}^{n+1} & =\left(I-\Delta t L^{-1} S\right) U^{n}+\Delta t L^{-1} F^{n}, \quad \text { and } \\
U_{j}^{n+1} & =\max \left(\widetilde{U}_{j}^{n+1}, \psi\left(P_{j}\right)\right) .
\end{aligned}
$$

3. The Truncation Method as a Perturbation of an $L^{2}$ Projection Scheme. The $L^{2}$ projection type method that we will consider (5) to be a perturbation of is (denoting its approximate solution vector by $W$ )

$$
\begin{aligned}
& \widetilde{W}^{n+1}=\left(I-\Delta t M^{-1} S\right) W^{n}+\Delta t M^{-1} F^{n}, \\
& W^{n+1}=L^{2}(\Omega) \text { projection of } \widetilde{W}^{n+1} \text { into } K_{n} .
\end{aligned}
$$

However, (as discussed in [9]) (6b) is equivalent to

$$
\left(W^{n+1}, V^{n+1}-W^{n+1}\right) \geqslant\left(\widetilde{W}^{n+1}, V^{n+1}-W^{n+1}\right) \text { for all } V^{n+1} \in K_{n},
$$

and so $W^{n+1} \in K_{h}$ is given by

$$
\begin{aligned}
& \left(W^{n+1}-W^{n}, V^{n+1}-W^{n+1}\right) / \Delta t+a\left(W^{n}, V^{n+1}-W^{n+1}\right) \\
& \geqslant\left(f^{n}, V^{n+1}-W^{n+1}\right) \text { for all } V^{n+1} \in K_{h} .
\end{aligned}
$$

For any $V, W \in S_{h}$ we define the lumped $L^{2}$ inner product

$$
(V, W)_{*}=V^{t} L W, \quad|V|_{L}^{2}=(V, V)_{*}
$$

and note that

$$
(V, W)_{*}=(V, W)+q(V, W) \text { for } V, W \in S_{h},
$$

where $q$ is the quadrature error. Analogous to (6)-(8), the truncation method (5) is precisely: find $U^{n+1}$ in $K_{n}$ such that

$$
\begin{aligned}
\left(U^{n+1}-U^{n}, V^{n+1}-U^{n+1}\right)_{*} / \Delta t+a\left(U^{n}, V^{n+1}-U^{n+1}\right) \\
\geqslant\left(f^{n}, V^{n+1}-U^{n+1}\right) \text { for all } V^{n+1} \in K_{n} .
\end{aligned}
$$

This follows using the definition of $\widetilde{U}^{n+1}$ to rewrite (11) as

$$
\left(U^{n+1}, V^{n+1}-U^{n+1}\right)_{*} \geqslant\left(\widetilde{U}^{n+1}, V^{n+1}-U^{n+1}\right)_{*} \text { for all } V^{n+1} \in K_{n} .
$$

Since $L$ is a diagonal matrix whose diagonal entries are positive, (12) gives (5b). But (11) has the form of (8) with the perturbation $q^{n+1}=q\left(U^{n+1}-U^{n}, V^{n+1}-U^{n+1}\right) / \Delta t$ added to the left side. Since an analysis of (8) is available [8], [6] (actually with $W^{n}, f^{n}$ replaced by $W^{n+1}, f^{n+1}$ in (8)-but this is not a major change), we are led to a line of attack on (11).

4. An Error Estimate for the Truncation Method. Before stating the result, we need one more bit of technical notation, regarding how rapidly the interface between where $u=\psi$ and $u>\psi$ shifts about. Fix $T>0$, and let $\Delta t=T / N$ for some positive integer $N$ (we assume $\Delta t \leqslant C_{1}$ ). As in [8], we let $\Omega^{+}(t)=\{p \in \Omega / u(p, t)>\psi(p)\}$. For $t \in\left(t^{n}, t^{n+1}\right]$; let $\Delta_{n}(t)$ be defined to be the symmetric difference of $\Omega^{+}\left(t^{n+1}\right)$ and $\Omega^{+}(t)$, and let $\delta_{n}(t)$ be the Lebesgue measure of $\Delta_{n}(t)$. Let $\delta(t)=\delta_{n}(t)$ when $t \in\left(t^{n}, t^{n+1}\right], n=0,1, \ldots, N-1$. We will assume that $\delta(t) \leqslant C_{2} \Delta t$ a.e. in $[0, T]$ for some constant $C_{2}$. This is generally more restrictive than the assumption in [8], 
thus permitting a more optimistic error estimate. Making the same assumption as [8] gives the same rate of convergence as in [8]. We let $\alpha$ denote the size of the smallest angle occurring in a triangular mesh and for any function $g$ we let $|g|^{2}=(g, g),\|g\|^{2}$ $=a(g, g)$. For our proof we also need to assume that $\alpha$ is independent of $h$ and that the ratio of the smallest mesh length to the largest mesh length is bounded above by some constant $\sigma>0$, which gives the inverse property

(13) $\|V\|^{2} \leqslant D h^{-2}|V|^{2} \quad$ for $V \in S_{h}$ (D is a constant depending only on $\alpha$ and $\sigma$ ).

This is easy to verify on any element of the mesh, and hence (13) follows by summing over the elements. Similarly, one observes that there are positive numbers $C_{3}$ and $C_{4}$ (independent of $\Omega$ and the mesh) such that

$$
C_{3}|V|_{L}^{2} \leqslant|V|^{2} \leqslant C_{4}|V|_{L}^{2} \quad \text { for } V \in S_{h}
$$

We have

THEOREM 1. In addition to the previous hypotheses, assume that the stability condition $\Delta t \leqslant h^{2} / 64 D$ is satisfied, and pick $U^{0}$ in $K_{h}$ so that $\left\|u^{0}-U^{0}\right\|=O(h)$. Let $\epsilon$ be a fixed number in $(0,1 / 2]$. Then for $u$ the solution of $(1), U$ the truncation method approximate solution (5), and $Z=u-U$,

$$
\begin{aligned}
E & \equiv \max _{n=1, \ldots, N}\left|Z^{n}\right|^{2}+\sum_{n=0}^{N-1}\left|Z^{n+1}-Z^{n}\right|^{2}+\sum_{n=1}^{N}\left\|Z^{n}\right\|^{2} \cdot \Delta t \\
& \leqslant C \cdot \epsilon^{-2} \cdot \Delta t^{2-2 \epsilon}+C h^{2} .
\end{aligned}
$$

We are using $C$ to denote a generic constant independent of $\Delta t, h, \epsilon$ but which may depend on $\Omega, u, f, T, \alpha, \sigma, C_{1}, C_{2}$. If $\Omega \subset R^{1}$, the epsilons can be omitted; and so the estimate becomes $E \leqslant C \Delta t^{2}+C h^{2}$.

We will outline the logic in a sequence of lemmas, and then give the proofs. The $\epsilon$ terms in (15) come from the necessity of estimating the terms

$$
\rho^{n} \equiv u_{t}^{n+1}-\left(u^{n+1}-u^{n}\right) / \Delta t .
$$

It is here that smoothness additional to the general a priori estimates seems to be required, otherwise there may be a $C \Delta t$ term on the right side of (15) [8]. Following the same kind of analysis and lengthy algebra as in [6], [8], and using results in [5], one obtains

LEMma 1.

$$
E \leqslant C \cdot \epsilon^{-2} \cdot \Delta t^{2-2 \epsilon}+C h^{2}+C \sum_{n=0}^{N-1}\left|q^{n+1}\right| \Delta t
$$

and if $\Omega \subset R^{1}$, the epsilons can be omitted.

Thus, we must estimate the $q^{n}$. We need only consider the quadrature error on elements $V, W$ of $S_{h}$, and letting $(V W)_{I}$ be the piecewise linear interpolate of $V W$,

$$
q(V, W)=\int_{\Omega}\left[V W-(V W)_{I}\right] d z
$$

and so by approximation theory, 


\section{LEMMA 2.}

$$
|q(V, W)| \leqslant D_{1} h^{2}\|V\|\|W\|, \quad \text { where } D_{1} \text { depends only on } \alpha .
$$

Thus from (17), we must consider

$$
E_{1} \equiv C \Delta t \sum_{n=0}^{N-1}\left|q^{n+1}\right| \leqslant C \Delta t \sum_{n=0}^{N-1} h^{2}\left\|\frac{U^{n+1}-U^{n}}{\Delta t}\right\|\left\|V^{n+1}-U^{n+1}\right\| .
$$

We first deal with $\left\|\left(U^{n+1}-U^{n}\right) / \Delta t\right\|$. Using (5), (13), (14), the hypothesis that $u^{0}$ $\in H^{2}(\Omega)$, the choice of $U^{0}$ to be such that $\left\|u^{0}-U^{0}\right\| \leqslant C h$, and the stability condition on $\Delta t$, one has

\section{LEMMA 3.}

$$
\Delta t \sum_{n=0}^{N-1}\left(\left|U^{n+1}-U^{n}\right| / \Delta t\right)^{2} \leqslant C
$$

Returning to (19) and using the arithmetic-geometric mean inequality $a b \leqslant a^{2} / 2 \rho$ $+\rho b^{2} / 2$, and $(a+b)^{2} \leqslant 2 a^{2}+2 b^{2}$ for $a, b, \rho \geqslant 0$ :

$$
E_{1} \leqslant C \Delta t \sum_{n=0}^{N-1} h^{4} \Delta t^{-2}\left\|U^{n+1}-U^{n}\right\|^{2}+\frac{\Delta t}{4} \sum_{n=0}^{N-1}\left\|V^{n+1}-U^{n+1}\right\|^{2},
$$

which by (13) and Lemma 3 gives

$$
E_{1} \leqslant C h^{2}+\frac{\Delta t}{4} \sum_{n=0}^{N-1}\left\|V^{n+1}-U^{n+1}\right\|^{2}
$$

$$
\leqslant C h^{2}+\frac{\Delta t}{2} \sum_{n=0}^{N-1}\left\|V^{n+1}-u^{n+1}\right\|^{2}+\frac{\Delta t}{2} \sum_{n=0}^{N-1}\left\|Z^{n+1}\right\|^{2} .
$$

Now (17) and (21) show that

$$
E \leqslant C \cdot \epsilon^{-2} \cdot \Delta t^{2-2 \epsilon}+C h^{2}+\Delta t \sum_{n=0}^{N-1}\left\|V^{n+1}-u^{n+1}\right\|^{2}
$$

and so Theorem 1 follows by choosing (as will be done in demonstrating Lemma 1) $V^{n+1}$ such that $\left\|V^{n+1}-u^{n+1}\right\| \leqslant C h$.

Proof of Lemma 1. We henceforth take $u_{t}$ to be $\partial^{+} u / \partial t$ (the right-hand derivative). Then by [3], Eq. (1b) is valid for every $t>0$. From (1b), (16), and (11) we have

$$
\begin{aligned}
\left(u^{n+1}-\right. & \left.u^{n}+\Delta t \cdot \rho^{n}, v^{n+1}-u^{n+1}\right) / \Delta t \\
& +a\left(u^{n+1}, v^{n+1}-u^{n+1}\right)+\left(U^{n+1}-U^{n}, V^{n+1}-U^{n+1}\right) / \Delta t \\
& +a\left(U^{n+1}, V^{n+1}-U^{n+1}\right)+q^{n+1}+p^{n+1} \\
\geqslant & \left(f^{n+1}, v^{n+1}-u^{n+1}\right)+\left(f^{n+1}, V^{n+1}-U^{n+1}\right)
\end{aligned}
$$

for all $v^{n+1} \in K, V^{n+1} \in K_{h}$, where 


$$
\begin{aligned}
p^{n+1}= & a\left(U^{n}-U^{n+1}, V^{n+1}-U^{n+1}\right) \\
& +\left(f^{n+1}-f^{n}, V^{n+1}-U^{n+1}\right) .
\end{aligned}
$$

Now (22) (with $q^{n+1}+p^{n+1}$ omitted) is (formally) the situation analyzed in [6] and [8], so we will follow the analysis there as closely as possible. By direct algebraic verification (noting that $a(v, w)$ is symmetric), one has that $(22)$ is equivalent to

$$
\begin{aligned}
& \left\{\left|Z^{n+1}\right|^{2}-\left(Z^{n+1}, Z^{n}\right)\right\} / \Delta t+\left\|Z^{n+1}\right\|^{2} \\
& \leqslant a\left(u^{n+1}, v^{n+1}-U^{n+1}\right)-\left(f^{n+1}, v^{n+1}-U^{n+1}\right) \\
& \quad+a\left(U^{n+1}, V^{n+1}-u^{n+1}\right) \\
& \quad-\left(f^{n+1}, V^{n+1}-u^{n+1}\right)+\left(\rho^{n}, v^{n+1}-u^{n+1}\right) \\
& \quad+\left(u^{n+1}-u^{n}, v^{n+1}-U^{n+1}\right) / \Delta t+\left(U^{n+1}-U^{n}, V^{n+1}-u^{n+1}\right) / \Delta t \\
& \quad+q^{n+1}+p^{n+1} .
\end{aligned}
$$

We let $A$ denote the bounded linear operator from $H^{2}(\Omega)$ into $L^{2}(\Omega)$ satisfying the integration by parts relation

$$
a(w, g)=(A w, g) \text { for } w \in H^{2}(\Omega), g \in H_{0}^{1}(\Omega) .
$$

Then operating separately on each side, (24) is equivalent to

$$
\begin{aligned}
&\left(\left|Z^{n+1}\right|^{2}-\left|Z^{n}\right|^{2}+\left|Z^{n+1}-Z^{n}\right|^{2}\right) / 2 \Delta t+\left\|Z^{n+1}\right\|^{2} \\
& \leqslant\left(u_{t}^{n+1}+A u^{n+1}-f^{n+1}, v^{n+1}-U^{n+1}+V^{n+1}-u^{n+1}\right) \\
&+a\left(Z^{n+1}, u^{n+1}-V^{n+1}\right)-\left(\rho^{n}, Z^{n+1}\right)-\left(\rho^{n}, V^{n+1}-u^{n+1}\right) \\
&+\left(Z^{n+1}-Z^{n}, u^{n+1}-V^{n+1}\right) / \Delta t+q^{n+1}+p^{n+1}
\end{aligned}
$$

We now choose $V^{n+1} \in K_{n}$ to be the approximation to $u^{n+1}$ used in [8] (in the notation of [8], $V^{n+1}=\left[S_{n}\left(u^{n+1}-\psi\right)\right]_{I}+\psi_{I}$, where $S_{h}$ is a smoothing function which keeps nonnegative functions nonnegative), so

$$
\left|V^{n+1}-u^{n+1}\right| \leqslant C h^{2} \text { and }\left\|V^{n+1}-u^{n+1}\right\| \leqslant C h .
$$

By Lemma 4 of [5], we may take $v^{n+1}(p) \equiv \max \left(U^{n+1}(p), \psi(p)\right)$ for $p \in \Omega$, and have $v^{n+1} \in K$ with $\left|v^{n+1}-U^{n+1}\right| \leqslant D_{2} h^{2}$ ( $D_{2}$ depending only on $\psi$ and $\alpha$ ). We also recall (e.g. [10]) that for some constant $D_{3}$ depending only on $\Omega,|w|^{2} \leqslant D_{3}\|w\|^{2}$ for $w \in H_{0}^{1}(\Omega)$.

Multiplying (26) by $2 \Delta t$ and summing from $n=0, \ldots, \widetilde{N}$ for each $0 \leqslant \widetilde{N}$ $\leqslant N-1$, we easily establish that 


$$
\begin{aligned}
E-2\left|Z^{0}\right|^{2} & \\
\leqslant 4 \Delta t \sum_{n=0}^{N-1}\{ & \left\{\left(u_{t}^{n+1}+A u^{n+1}-f^{n+1}, v^{n+1}-U^{n+1}+V^{n+1}-u^{n+1}\right) \mid\right. \\
& +\left|a\left(Z^{n+1}, u^{n+1}-V^{n+1}\right)\right|+\left|\left(\rho^{n}, Z^{n+1}\right)\right| \\
& +\left|\left(\rho^{n}, V^{n+1}-u^{n+1}\right)\right|+\left|\left(Z^{n+1}-Z^{n}, u^{n+1}-V^{n+1}\right) / \Delta t\right| \\
& \left.+\left|q^{n+1}\right|+\left|p^{n+1}\right|\right\} .
\end{aligned}
$$

Applying the approximation results and the arithmetic-geometric mean inequality, the first two terms on the right side of (27) can be bounded by $\mathrm{Ch}^{2}$ and $\mathrm{Ch}^{2}+$ $\Delta t \Sigma_{n=0}^{N-1}\left\|Z^{n+1}\right\|^{2} / 16$, respectively.

The next two expressions can be bounded by

$$
\Delta t \sum_{n=0}^{N-1}\left\|Z^{n+1}\right\|^{2} / 16+C \Delta t \sum_{n=0}^{N-1} \sup _{\|w\|=1}\left|\left(\rho^{n}, w\right)\right|^{2}+C \Delta t \sum_{n=0}^{N-1}\left|\rho^{n}\right| h^{2} .
$$

The expression

$$
4 \sum_{n=0}^{N-1}\left|\left(Z^{n+1}-Z^{n}, u^{n+1}-V^{n+1}\right)\right|
$$

is shown in [8] to be bounded by

$$
C h^{2}+\left|Z^{N}\right|^{2} / 8+\Delta t \sum_{n=1}^{N}\left\|Z^{n}\right\|^{2} / 8
$$

From the definition of $p^{n+1}$ and the arithmetic-geometric mean inequality we obtain

$$
\begin{aligned}
\left|p^{n+1}\right| \leqslant & 32\left\|U^{n}-U^{n+1}\right\|^{2} / 9+9\left\|V^{n+1}-U^{n+1}\right\|^{2} / 128 \\
& +C\left|f^{n+1}-f^{n}\right|^{2}+\left|V^{n+1}-U^{n+1}\right|^{2} / 128 D_{3} \\
\leqslant & 64 D h^{-2}\left|U^{n}-V^{n}+V^{n+1}-U^{n+1}\right|^{2} / 9 \\
& +C\left\|V^{n}-V^{n+1}\right\|^{2}+C\left\|V^{n+1}-u^{n+1}\right\|^{2} \\
& +5\left\|Z^{n+1}\right\|^{2} / 32+C\left|f^{n+1}-f^{n}\right|^{2} \\
\leqslant & 128 D h^{-2}\left|Z^{n+1}-Z^{n}\right|^{2} / 9+C h^{-2}\left|u^{n}-V^{n}\right|^{2} \\
& +C h^{-2}\left|u^{n+1}-V^{n+1}\right|^{2}+C\left\|V^{n}-u^{n}\right\|^{2} \\
& +C\left\|u^{n}-u^{n+1}\right\|^{2}+C\left\|V^{n+1}-u^{n+1}\right\|^{2} \\
& +5\left\|Z^{n+1}\right\|^{2} / 32+C\left|f^{n+1}-f^{n}\right|^{2}
\end{aligned}
$$

Thus, using the stability condition on $\Delta t$, 


$$
\begin{aligned}
4 \Delta t \sum_{n=0}^{N-1}\left|p^{n+1}\right| \leqslant & 8 \sum_{n=0}^{N-1}\left|Z^{n+1}-Z^{n}\right|^{2} / 9+C h^{2}+5 \Delta t \sum_{n=1}^{N}\left\|Z^{n}\right\|^{2} / 8 \\
& +C \Delta t^{2}\left\{\left\|f_{t}\right\|_{L^{2}\left(0, T, L^{2}(\Omega)\right)}^{2}+\left\|u_{t}\right\|_{L^{2}\left(0, T, H_{0}^{1}(\Omega)\right)}^{2}\right\} .
\end{aligned}
$$

The further estimation of the terms involving $\rho^{n}$ follows the method of analysis in [8]. The only (small) difference involves the use of our hypothesis on $\delta(t)$ for which the following lemma is convenient.

Lemma 4. Let $H \in L^{\infty}(\Omega), w \in H_{0}^{1}(\Omega), S$ a (Lebesgue) measurable subset of $\Omega, \sigma=$ measure $(S)$. Let $\epsilon$ be in $(0,1 / 2]$. Then for some constant $D_{4}$ depending only on $\Omega$,

$$
J \equiv\left|\int_{S} H(z) w(z) d z\right| \leqslant D_{4} \epsilon^{-1}\|H\|_{L^{\infty}(\Omega)} \sigma^{1-\epsilon}\|w\|
$$

Proof. From [10], if $q=\epsilon^{-1}$, then $w \in L^{q}(\Omega)$ and $\|w\|_{L^{q}(\Omega)} \leqslant D_{4} \epsilon^{-1}\|w\|$. Let $p=q /(q-1)$. Then $J \leqslant\|H\|_{L^{p}(S)}\|w\|_{L^{q}(\Omega)}$ and the result quickly follows. In the case $\Omega \subset R^{1}$, the result holds with the epsilons omitted, since then $H_{0}^{1}(\Omega) \subset$ $C^{0}(\bar{\Omega})$.

Using Lemma 4 with $S=\Delta_{n}(t)$ within the analysis in [8] results in

$$
\Delta t \sum_{n=0}^{N-1} \sup _{\|w\|=1}\left|\left(\rho^{n}, w\right)\right|^{2} \leqslant C \Delta t^{2}+C \epsilon^{-2} \int_{0}^{T} \delta^{2-2 \epsilon}(t) d t
$$

Finally, we observe that $\left|\rho^{n}\right| \leqslant C$ since

$$
\rho^{n}=\int_{t^{n}}^{t^{n+1}}\left(u_{t}^{n+1}-u_{t}(s)\right)(\Delta t)^{-1} d s
$$

and $u_{t} \in L^{\infty}\left(0, T, L^{\infty}(\Omega)\right)$. Combining all these results and recalling the hypothesis $\delta(t) \leqslant C_{2} \Delta t$ a.e. in $[0, T]$ completes the proof of Lemma 1 .

Proof of Lemma 2. From approximation theory (e.g. [11]) on each triangle (or interval) $e_{i}$,

$$
\left\|V W-(V W)_{I}\right\|_{L^{\infty}\left(e_{i}\right)} \leqslant \bar{D}_{1} h^{2} \max _{\beta_{1}+\beta_{2}=2}\left\|\frac{\partial^{\beta_{1}}}{\partial x^{\beta_{1}}} \frac{\partial^{\beta_{2}}}{\partial y^{\beta_{2}}}(V W)\right\|_{L^{\infty}\left(e_{i}\right)},
$$

where $\bar{D}_{1}$ is a constant depending only on $\alpha$ (and reference to $y$ is to be omitted in 1 space dimension). Since $V$ and $W$ are linear functions on $e_{i}$, this implies that

$$
\left|\int_{e_{i}}\left[(V W)_{I}-(V W)\right] d z\right| \leqslant 2 \bar{D}_{1} h^{2} \int_{e_{i}}\left(\left|V_{x} W_{x}\right|+\left|V_{y} W_{y}\right|+\left|V_{x} W_{y}\right|+\left|V_{y} W_{x}\right|\right) d z
$$

and so summing over the elements yields the result.

Proof of Lemma 3. Equation (5a) gives $\left(\widetilde{U}^{1}-U^{0}, V\right)_{*} / \Delta t+a\left(U^{0}, V\right)=$ $\left(f^{0}, V\right)$ for $V \in S_{h}$, from which, using (14), (25), and (13), there results 


$$
\begin{aligned}
\left|\left(\widetilde{U}^{1}-U^{0}\right) / \Delta t\right|_{L} & \leqslant \sup _{|V|_{L}=1}\left|a\left(U^{0}, V\right)\right|+\sqrt{C_{4}}\left|f^{0}\right| \\
& \leqslant \sqrt{C_{4}}\left(\sqrt{D} h^{-1}\left\|u^{0}-U^{0}\right\|+\left|A u^{0}\right|+\left|f^{0}\right|\right) .
\end{aligned}
$$

By hypothesis, $u^{0} \in H^{2}(\Omega)$, and $\left\|u^{0}-U^{0}\right\| \leqslant C h$, so by noting, from (5b), that $\left|U^{1}-U^{0}\right|_{L} \leqslant\left|\widetilde{U}^{1}-U^{0}\right|_{L}$, one has $\left|U^{1}-U^{0}\right|_{L} \leqslant D_{0} \Delta t$ for some sufficiently large $D_{0}$. Now using Eqs. (26) and (27) of [1], along with (13) shows that when $\Delta t \leqslant$ $2 h^{2} / D$,

$$
\left|\left(I-\Delta t L^{-1} S\right) V\right|_{L} \leqslant|V|_{L} \quad \text { for } V \in S_{h}
$$

By (5b)

$$
\left|U^{n+1}-U^{n}\right|_{L} \leqslant\left|\widetilde{U}^{n+1}-\widetilde{U}^{n}\right|_{L}
$$

Thus by (5a) and (28),

$$
\left|U^{n+1}-U^{n}\right|_{L} \leqslant\left|U^{n}-U^{n-1}\right|_{L}+\Delta t\left|L^{-1}\left(F^{n}-F^{n-1}\right)\right|_{L}
$$

Now using the mean value theorem, and recalling that $F_{j}^{n}=\left(\varphi_{j}, f(\cdot, n \Delta t)\right)$, it follows easily that $\left|F_{j}^{n}-F_{j}^{n-1}\right| \leqslant D_{5} h^{k} \Delta t$ for $j=1, \ldots, m$, where $k=1$ in $R^{1}, k=2$ in $R^{2}$, and $D_{5}$ depends only on $f_{t}$. Also, $m$ is bounded by three times the number of elements which is bounded by $D_{6} h^{-k}$ (where $D_{6}$ depends only on $\alpha, \sigma$, and the length or area of $\Omega$ ). Since $L^{-1}$ is a diagonal matrix whose elements are bounded above by $D_{7} h^{-k}\left(D_{7}\right.$ depending only on $\alpha$ and $\sigma$ ), one then obtains

$$
\Delta t\left|L^{-1}\left(F^{n}-F^{n-1}\right)\right|_{L} \leqslant D_{8} \Delta t^{2},
$$

where $D_{8}=D_{5} \sqrt{D_{6} D_{7}}$. Using (29) and (30), we have (by induction);

$$
\left|U^{n+1}-U^{n}\right|_{L} \leqslant D_{0} \Delta t+n D_{8} \Delta t^{2}
$$

and then (14) completes the proof.

Mathematical Analysis Branch

Code WA44

Naval Surface Weapons Center

Silver Spring, Maryland 20910

Department of Mathematics

Rutgers University

New Brunswick, New Jersey 08903

1. A. E. BERGER, "The truncation method for the numerical solution of variational inequalities," Rev. Française Automat. Informat. Recherche Opérationelle Sér. Rouge Anal. Numér., v. 10, 1976, pp. $29-42$.

2. A. E. BERGER, M. CIMENT \& J. C. W. ROGERS, "Numerical solution of a diffusion consumption problem with a free boundary," SIAM J. Numer. Anal., v. 12, 1975, pp. 646-672. MR 52 \# 4659.

3. H. BRÉZIS, "Problèmes unilateraux," J. Math. Pures Appl., v. 51, 1972, pp. 1-168.

4. G. DUVAUT \& J. L. LIONS, Les Inéquations en Mécanique et en Physique, Dunod, Paris, 1972.

5. R. S. FALK, "Error estimates for the approximation of a class of variational inequalities," Math. Comp., v. 28, 1974, pp. 963-971. MR 52 \#12323. 
6. R. FALK, Unpublished manuscript.

7. C. HUNT \& N. NASSIF, "Inéquations variationnelles et détermination de la charge d'espace de certaines semi-conducteurs," C. R. Acad. Sci. Paris Sér. A, v. 278, 1974, pp. 14091412. MR 49 \# 8509.

8. C. JOHNSON, "A convergence estimate for an approximation of a parabolic variational inequality," SIAM J. Numer. Anal., v. 13, 1976, pp. 599-606.

9. J. L. LIONS, “Approximation numérique des inéquations d'évolution," Constructive Aspects of Functional Analysis (C. I. M. E., II, Ciclo, 1971), G. Geymonat (Editor), Edizioni Cremonese, Rome, 1973.

10. J. NEČAS, Les Méthodes Directes en Théorie des Équations Elliptiques, Masson, Paris; Academia, Prague, 1967. MR 37 \# 3168.

11. G. STRANG, "Approximation in the finite element method," Numer. Math., v. 19, 1972, pp. 81-98. MR $46 \# 4677$. 